\title{
Critical Groups of Simplicial Complexes
}

\author{
Art M. Duval ${ }^{1}$, Caroline J. Klivans ${ }^{2}$ and Jeremy L. Martin ${ }^{3}$ \\ ${ }^{1}$ Department of Mathematical Sciences, University of Texas at El Paso, TX, USA \\ ${ }^{2}$ Departments of Mathematics and Computer Science, The University of Chicago, IL, USA \\ ${ }^{3}$ Department of Mathematics, University of Kansas, KS, USA
}

\begin{abstract}
We generalize the theory of critical groups from graphs to simplicial complexes. Specifically, given a simplicial complex, we define a family of abelian groups in terms of combinatorial Laplacian operators, generalizing the construction of the critical group of a graph. We show how to realize these critical groups explicitly as cokernels of reduced Laplacians, and prove that they are finite, with orders given by weighted enumerators of simplicial spanning trees. We describe how the critical groups of a complex represent flow along its faces, and sketch another potential interpretation as analogues of Chow groups.

Résumé. Nous généralisons la théorie des groupes critiques des graphes aux complexes simpliciaux. Plus précisément, pour un complexe simplicial, nous définissons une famille de groupes abéliens en termes d'opérateurs de Laplace combinatoires, qui généralise la construction du groupe critique d'un graphe. Nous montrons comment réaliser ces groupes critiques explicitement comme conoyaux des opérateurs de Laplace réduits combinatoires, et montrons qu'ils sont finis. Leurs ordres sont obtenus en comptant (avec des poids) des arbres simpliciaux couvrants. Nous décrivons comment les groupes critiques d'un complexe représentent le flux le long de ses faces, et esquissons une autre interprétation potentielle comme analogues des groupes de Chow.
\end{abstract}

Keywords: graph, simplicial complex, critical group, combinatorial Laplacian, chip-firing game, sandpile model, spanning trees

\section{Introduction}

Let $G$ be a finite, simple, undirected, connected graph. The critical group of $G$ is a finite abelian group $K(G)$ whose cardinality is the number of spanning trees of $G$. The critical group is an interesting graph invariant in its own right, and it also arises naturally in the theory of a discrete dynamical system with many essentially equivalent formulations — the chip-firing game, dollar game, abelian sandpile model, etc.that has been discovered independently in contexts including statistical physics, arithmetic geometry, and combinatorics. There is an extensive literature on these models and their behavior: see, e.g., [3, 4, 7, 14, [20]. In all guises, the model describes a certain type of discrete flow along the edges of $G$. The elements of the critical group correspond to states in the flow model that are stable, but for which a small perturbation causes an instability.

The purpose of this paper is to extend the theory of the critical group from graphs to simplicial complexes. For a finite simplicial complex $\Delta$ of dimension $d$, we define its higher critical groups as

$$
K_{i}(\Delta):=\operatorname{ker} \partial_{i} / \operatorname{im}\left(\partial_{i+1} \partial_{i+1}^{*}\right)
$$

1365-8050 @ 2011 Discrete Mathematics and Theoretical Computer Science (DMTCS), Nancy, France 
for $0 \leq i \leq d-1$; here $\partial_{j}$ means the simplicial boundary map mapping $j$-chains to $(j-1)$-chains. The map $\partial_{i+1} \partial_{i+1}^{*}$ is called an (updown) combinatorial Laplacian operator. For $i=0$, our definition coincides with the standard definition of the critical group of the 1-skeleton of $\Delta$. Our main result (Theorem 3.4 states that, under certain mild assumptions on the complex $\Delta$, the group $K_{i}(\Delta)$ is in fact isomorphic to the cokernel of a reduced version of the Laplacian. It follows from a simplicial analogue of the matrix-tree theorem [9, 10] that the orders $\left|K_{i}(\Delta)\right|$ of the higher critical groups are given by a torsion-weighted enumeration of higher-dimensional spanning trees (Corollary 4.2 ) and in terms of the eigenvalues of the Laplacian operators (Corollary 4.4p. In the case of a simplicial sphere, we prove (Theorem 4.6 that the top-dimensional critical group is cyclic, with order equal to the number of facets, generalizing the corresponding statement [21, 22, 24] for cycle graphs. In the case that $\Delta$ is a skeleton of an $n$-vertex simplex, the critical groups are direct sums of copies of $\mathbb{Z} / n \mathbb{Z}$; as we discuss in Remark 4.7. this follows from an observation of Maxwell [23] together with our main result. We also give a model of discrete flow (Section 5) on the codimension-one faces along facets of the complex whose behavior is captured by the group structure. Finally, we outline (Section 6) an alternative interpretation of the higher critical groups as discrete analogues of the Chow groups of an algebraic variety.

The authors thank Andy Berget, Hailong Dao, Craig Huneke, Manoj Kummini, Gregg Musiker, Igor Pak, Vic Reiner, and Ken Smith for numerous helpful discussions.

In this extended abstract, we omit the proofs; they can be found in the full version of the article [11].

\section{Critical Groups of Graphs}

\subsection{The chip-firing game}

We summarize the chip-firing game on a graph, omitting the proofs. For more details, see, e.g., Biggs [3].

Let $G=(V, E)$ be a finite, simple $\left.{ }^{\text {(i) }}\right)$ connected, undirected graph, with $V=[n] \cup q=\{1,2, \ldots, n, q\}$ and $E=\left\{e_{1}, \ldots, e_{m}\right\}$. The special vertex $q$ is called the bank (or "root" or "government"). Let $d_{i}$ be the degree of vertex $i$, i.e. the number of adjacent vertices. The chip-firing game is a discrete dynamical system whose state is described by a configuration vector $\mathbf{c}=\left(c_{1}, \ldots, c_{n}\right) \in \mathbb{N}^{n}$. Each $c_{i}$ is a nonnegative integer that we think of as the number of "chips" belonging to vertex $i$. (Note that the number $c_{q}$ of chips belonging to the bank $q$ is not part of the data of a configuration.)

Each non-root vertex is generous (it likes to donate chips to its neighbors), egalitarian (it likes all its neighbors equally), and prudent (it does not want to go into debt). Specifically, a vertex $v_{i}$ is called ready in a configuration $\mathbf{c}$ if $c_{i} \geq d_{i}$. If a vertex is ready, it can fire by giving one chip to each of its neighbors. Unlike the other vertices, the bank is a miser. As long as other vertices are firing, the bank does not fire, but just collects chips.

As more and more chips accumulate at the bank, the game eventually reaches a configuration in which no non-bank vertex can fire. Such a configuration is called stable. At this point, the bank finally fires, giving one chip to each of its neighbors. Unlike the other vertices, the bank is allowed to go into debt: that is, we do not require that $c_{q} \geq d_{q}$ for the bank to be able to fire.

Denote by $\mathbf{c}\left(x_{1}, \ldots, x_{r}\right)$ the configuration obtained from $\mathbf{c}$ by firing the vertices $x_{1}, \ldots, x_{r}$ in order. This sequence (which may contain repetitions) is called a firing sequence for $\mathbf{c}$ if every firing is permissible: that is, for each $i$, either $x_{i} \neq q$ is ready to fire in the configuration $\mathbf{c}\left(x_{1}, \ldots, x_{i-1}\right)$, or else $x_{i}=q$

(i) The chip-firing game and our ensuing results can easily be extended to allow parallel edges; we assume that $G$ is simple for the sake of ease of exposition. 
and $\mathbf{c}\left(x_{1}, \ldots, x_{i-1}\right)$ is stable. A configuration $\mathbf{c}$ is called recurrent if there is a nontrivial firing sequence $X$ such that $\mathbf{c}(X)=\mathbf{c}$.

A configuration is called critical if it is both stable and recurrent. For every starting configuration c, there is a uniquely determined critical configuration $[\mathbf{c}]$ that can be reached from $\mathbf{c}$ by some firing sequence [3, Thm. 3.8]. The critical group $K(G)$ is defined as the set of these critical configurations, with group law given by $[\mathbf{c}]+\left[\mathbf{c}^{\prime}\right]=\left[\mathbf{c}+\mathbf{c}^{\prime}\right]$, where the right-hand addition is componentwise addition of vectors.

The abelian sandpile model was first introduced in [7] as an illustration of "self-organized criticality"; an excellent recent exposition is [20]. Here, grains of sand (analogous to chips) are piled at each vertex, and an additional grain of sand is added to a (typically randomly chosen) pile. If the pile reaches some predetermined size (for instance, the degree of that vertex), then it topples by giving one grain of sand to each of its neighbors, which can then topple in turn, and so on. This sequence of topplings is called an avalanche and the associated operator on states of the system is called an avalanche operator. (One can show that the avalanche operator does not depend on the order in which vertices topple; this is the reason for the use of the term "abelian".) The sandpile model itself is the random walk on the stable configurations, and the critical group is the group generated by the avalanche operators.

The critical group can also be viewed as a discrete analogue of the Picard group of an algebraic curve. This point of view goes back at least as far as the work of Lorenzini [21, 22] and was developed, using the language of divisors, by Bacher, de la Harpe, and Nagnibeda [1] (who noted that their "setting has a straightforward generalization to higher dimensional objects"). It appears in diverse combinatorial contexts including elliptic curves over finite fields (Musiker [25]), linear systems on tropical curves (Haase, Musker and $\mathrm{Yu}$ [15]), and Riemann-Roch theory for graphs (Baker and Norine [2]).

\subsection{The algebraic viewpoint}

The critical group can be defined algebraically in terms of the Laplacian matrix.

Definition 2.1 Let $G$ be a finite, simple, connected, undirected graph with vertices $\{1, \ldots, n, q\}$. The Laplacian matrix of $G$ is the symmetric matrix $L$ (or, equivalently, linear self-adjoint operator) whose rows and columns are indexed by the vertices of $G$, with entries

$$
\ell_{i j}= \begin{cases}d_{i} & \text { if } i=j, \\ -1 & \text { if } i j \in E \\ 0 & \text { otherwise }\end{cases}
$$

Firing vertex $i$ in the chip-firing game is equivalent to subtracting the $i^{t h}$ column of the Laplacian (ignoring the entry indexed by $q$ ) from the configuration vector $\mathbf{c}$. Equivalently, if $\mathbf{c}^{\prime}=\mathbf{c}\left(x_{1}, \ldots, x_{r}\right)$, then the configurations $\mathbf{c}$ and $\mathbf{c}^{\prime}$ represent the same element of the cokernel of the Laplacian (that is, the quotient of $\mathbb{Z}^{n+1}$ by the column space of $L$ ).

It is immediate from the definition of $L$ that $L(\mathbf{1})=\mathbf{0}$, where $\mathbf{1}$ and $\mathbf{0}$ denote the all-ones and all-zeros vectors in $\mathbb{N}^{n+1}$. Moreover, it is not difficult to show that $\operatorname{rank} L=|V|-1=n$. In terms of homological algebra, we have a chain complex

$$
\mathbb{Z}^{n+1} \stackrel{L}{\rightarrow} \mathbb{Z}^{n+1} \stackrel{S}{\rightarrow} \mathbb{Z} \rightarrow 0
$$

where $S(\mathbf{c})=\mathbf{c} \cdot \mathbf{1}=c_{q}+c_{1}+\cdots+c_{n}$. The equation $L(\mathbf{1})=\mathbf{0}$ says that $\operatorname{ker}(S) \supseteq \operatorname{im}(L)$. Moreover, $\operatorname{rank} L=n=\operatorname{rank} \operatorname{ker} S$, so the abelian group $\operatorname{ker}(S) / \operatorname{im}(L)$ is finite. 
Definition 2.2 The critical group of a graph $G$ is $K(G)=\operatorname{ker}(S) / \operatorname{im}(L)$.

This definition of the critical group is equivalent to that in terms of the chip-firing game [3, Thm. 4.2]. The order of the critical group is the determinant of the reduced Laplacian formed by removing the row and column indexed by $q[3$, Thm. 6.2]. By the matrix-tree theorem, this is the number of spanning trees. As we will see, the algebraic description provides a natural framework for generalizing the critical group.

\section{The Critical Groups of a Simplicial Complex}

We assume familiarity with the basic algebraic topology of simplicial complexes; see, e.g., Hatcher [17]. Let $\Delta$ be a $d$-dimensional simplicial complex. For $-1 \leq i \leq d$, let $C_{i}(\Delta ; \mathbb{Z})$ be the $i^{\text {th }}$ simplicial chain group of $\Delta$. We denote the simplicial boundary and coboundary maps respectively by

$$
\begin{aligned}
& \partial_{\Delta, i}: C_{i}(\Delta ; \mathbb{Z}) \rightarrow C_{i-1}(\Delta ; \mathbb{Z}), \\
& \partial_{\Delta, i}^{*}: C_{i-1}(\Delta ; \mathbb{Z}) \rightarrow C_{i}(\Delta ; \mathbb{Z}),
\end{aligned}
$$

where we have identified cochains with chains via the natural inner product. We will abbreviate the subscripts in the notation for boundaries and coboundaries whenever no ambiguity can arise.

Let $-1 \leq i \leq d$. The $i$-dimensional combinatorial Laplacian ${ }^{\text {(ii) }}$ of $\Delta$ is the operator

$$
L_{\Delta, i}=\partial_{i+1} \partial_{i+1}^{*}: C_{i}(\Delta ; \mathbb{Z}) \rightarrow C_{i}(\Delta ; \mathbb{Z}) .
$$

Combinatorial Laplacian operators seem to have first appeared in the work of Eckmann [12] on finite dimensional Hodge theory. As the name suggests, they are discrete versions of the Laplacian operators on differential forms on a Riemannian manifold. In fact, Dodziuk and Patodi [8] showed that for suitably nice triangulations of a manifold, the eigenvalues of the discrete Laplacian converge in an appropriate sense to those of the usual continuous Laplacian. For one-dimensional complexes, i.e., graphs, the combinatorial Laplacian is just the usual Laplacian matrix $L=D-A$, where $D$ is the diagonal matrix of vertex degrees and $A$ is the (symmetric) adjacency matrix.

In analogy to the chain complex of (1), we have the chain complex

$$
C_{i}(\Delta ; \mathbb{Z}) \stackrel{L}{\rightarrow} C_{i}(\Delta ; \mathbb{Z}) \stackrel{\partial_{i}}{\longrightarrow} C_{i-1}(\Delta ; \mathbb{Z})
$$

where $L=L_{\Delta, i}$. (This is a chain complex because $\partial_{i} L=\partial_{i} \partial_{i+1} \partial_{i+1}^{*}=0$.) We are now ready to make our main definition.

Definition 3.1 The i-dimensional critical group of $\Delta i s$

$$
K_{i}(\Delta):=\operatorname{ker} \partial_{i} / \operatorname{im} L=\operatorname{ker} \partial_{i} / \operatorname{im}\left(\partial_{i+1} \partial_{i+1}^{*}\right)
$$

Note that $K_{0}(\Delta)$ is precisely the critical group of the 1-skeleton of $\Delta$.

\footnotetext{
(ii) In other settings, our Laplacian might be referred to as the "up-down" Laplacian, $L^{\text {ud }}$. The $i^{\text {th }}$ down-up Laplacian is $L_{i}^{\text {du }}=$ $\partial_{i}^{*} \partial_{i}$, and the $i^{\text {th }}$ total Laplacian is $L_{i}^{\text {tot }}=L_{i}+L_{i}^{\text {du }}$. We adopt the notation we do since, except for one application (Remark 4.7 below), we only need the up-down Laplacian.
} 


\subsection{Simplicial spanning trees}

Our results about critical groups rely on the theory of simplicial and cellular spanning trees developed in [9], based on earlier work of Bolker [5] and Kalai [19]. Here we briefly review the definitions and basic properties, including the higher-dimensional analogues of Kirchhoff's matrix-tree theorem. For simplicity, we present the theory for simplicial complexes, the case of primary interest in combinatorics. Nevertheless, the definitions of spanning trees, their enumeration using a generalized matrix-tree theorem, and the definition and main result about critical groups are all valid in the more general setting of regular CW-complexes [10].

In order to define simplicial spanning trees, we first fix some notation concerning simplicial complexes and algebraic topology. The symbol $\Delta_{i}$ will denote the set of cells of dimension $i$. The $i$-dimensional skeleton $\Delta_{(i)}$ of a simplicial complex $\Delta$ is the subcomplex consisting of all cells of dimension $\leq i$. A complex is pure if all maximal cells have the same dimension. The $i^{t h}$ reduced homology group of $\Delta$ with coefficients in a ring $R$ is denoted $\tilde{H}_{i}(\Delta ; R)$. The Betti numbers of $\Delta$ are $\beta_{i}(\Delta)=\operatorname{dim}_{\mathbb{Q}} \tilde{H}_{i}(\Delta ; \mathbb{Q})$. The $f$-vector is $f(\Delta)=\left(f_{-1}(\Delta), f_{0}(\Delta), \ldots\right)$, where $f_{i}(\Delta)$ is the number of faces of dimension $i$.

Definition 3.2 Let $\Delta$ be a pure d-dimensional simplicial complex, and let $\Upsilon \subseteq \Delta$ be a subcomplex such that $\Upsilon_{(d-1)}=\Delta_{(d-1)}$. We say that $\Upsilon$ is a (simplicial) spanning tree of $\Delta$ if the following three conditions hold:

1. $\tilde{H}_{d}(\Upsilon ; \mathbb{Z})=0$;

2. $\tilde{H}_{d-1}(\Upsilon ; \mathbb{Q})=0$ (equivalently, $\left.\left|\tilde{H}_{d-1}(\Upsilon ; \mathbb{Z})\right|<\infty\right)$;

3. $f_{d}(\Upsilon)=f_{d}(\Delta)-\beta_{d}(\Delta)+\beta_{d-1}(\Delta)$.

More generally, an $i$-dimensional spanning tree of $\Delta$ is a spanning tree of the $i$-dimensional skeleton of $\Delta$.

In the case $d=1$ (that is, $\Delta$ is a graph), we recover the usual definition of a spanning tree: the three conditions above say respectively that $\Upsilon$ is acyclic, connected, and has one more vertex than edge. Meanwhile, the 0 -dimensional spanning trees of $\Delta$ are its vertices (more precisely, the subcomplexes of $\Delta$ with a single vertex), which are precisely the connected, acyclic subcomplexes of $\Delta_{(0)}$.

Just as in the graphical case, any two of the conditions of Definition 3.2 imply the third [9, Prop 3.5]. In order for $\Delta$ to have a $d$-dimensional spanning tree, it is necessary and sufficient that $\tilde{H}_{i}(\Delta ; \mathbb{Q})=0$ for all $i<d$; such a complex is called acyclic in positive codimension, or APC. Note that a graph is APC if and only if it is connected.

Example 3.3 Consider the equatorial bipyramid: the two-dimensional simplicial complex $B$ with vertices [5] and facets 123, 124, 125, 134, 135, 234, 235. A geometric realization of $B$ is shown in Figure 1] A 2SST of $B$ can be constructed by removing two facets $F, F^{\prime}$, provided that $F \cap F^{\prime}$ contains neither of the vertices 4,5 . A simple count shows that there are 15 such pairs $F, F^{\prime}$, so $B$ has 15 two-dimensional spanning trees.

A phenomenon arising only in dimension $d>1$ is that spanning trees may have torsion: that is, $\tilde{H}_{d-1}(\Upsilon ; \mathbb{Z})$ can be finite but nontrivial. For example, the 2-dimensional skeleton of a 6-vertex simplex has (several) spanning trees $\Upsilon$ that are homeomorphic to the real projective plane, and in particular have $\tilde{H}_{1}(\Upsilon ; \mathbb{Z}) \cong \mathbb{Z} / 2 \mathbb{Z}$. This cannot happen in dimension 1 (i.e., for graphs), in which every spanning tree is 


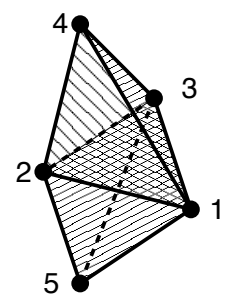

Fig. 1: The equatorial bipyramid $B$.

a contractible topological space. This torsion directly affects tree enumeration in higher dimension; see Section 4

\subsection{The main theorem}

Our main result gives an explicit form for the critical group $K_{i}(\Delta)$ in terms of a reduced Laplacian matrix. This reduced form is both more convenient for computing examples, and gives a direct connection with the simplicial and cellular generalizations of the matrix-tree theorem [9, 10].

Let $\Delta$ be a pure, $d$-dimensional, APC simplicial complex, and fix $i<d$. Let $\Upsilon$ be an $i$-dimensional spanning tree of $\Delta_{(i)}$, and let $\Theta=\Delta_{i} \backslash \Upsilon$ (the set of $i$-dimensional faces of $\Delta$ not in $\Upsilon$ ). Let $\tilde{L}$ denote the reduced Laplacian obtained from $L$ by removing the rows and columns corresponding to $\Upsilon$ (equivalently, by restricting $L$ to the rows and columns corresponding to $\Theta$ ).

Theorem 3.4 Suppose that $\tilde{H}_{i-1}(\Upsilon ; \mathbb{Z})=0$. Then

$$
K_{i}(\Delta) \cong \mathbb{Z}^{\Theta} / \operatorname{im} \tilde{L}
$$

We omit the proof, which uses some basic homological algebra; for details, see [11].

Example 3.5 We return to the bipyramid B from Example 3.3 to illustrate Theorem 3.4 We must first pick a 1-dimensional spanning tree $\Upsilon$; we take $\Upsilon$ to be the spanning tree with edges $12,13,14,15$. (In general, we must also make sure $\Upsilon$ is torsion-free, but this is always true for 1-dimensional trees.) Let $L=L_{B, 1}: C_{1}(B ; \mathbb{Z}) \rightarrow C_{1}(B ; \mathbb{Z})$ be the full Laplacian; note that $L$ is a $9 \times 9$ matrix whose rows and columns are indexed by the edges of $B$. The reduced Laplacian $\tilde{L}$ is formed by removing the rows and columns indexed by the edges of $\Upsilon$ :

$$
\tilde{L}=\begin{array}{rcccc}
23 & 24 & 25 & 34 & 35 \\
23 & 24 \\
25 & 34 \\
35
\end{array}\left(\begin{array}{ccccc}
3 & -1 & -1 & 1 & 1 \\
-1 & 2 & 0 & -1 & 0 \\
-1 & 0 & 2 & 0 & -1 \\
1 & -1 & 0 & 2 & 0 \\
1 & 0 & -1 & 0 & 2
\end{array}\right) .
$$

The critical group $K_{1}(B)$ is the cokernel of this matrix, i.e., $K_{1}(B) \cong \mathbb{Z}^{5} /$ im $\tilde{L}$. Since $\tilde{L}$ has full rank, it follows that $K_{1}(B)$ is finite; its order is $\operatorname{det}(\tilde{L})=15$. 


\section{The Order of the Critical Group}

The matrix-tree theorem implies that the order of the critical group of a graph equals the number of spanning trees. In this section, we explain how this equality carries over to the higher-dimensional setting.

As before, let $\Delta$ be a pure $d$-dimensional simplicial complex. Let $\mathcal{T}_{i}(\Delta)$ denote the set of all $i$ dimensional spanning trees of $\Delta$ (that is, spanning trees of the $i$-dimensional skeleton $\Delta_{(i)}$ ). Define

$$
\begin{aligned}
\tau_{i} & =\sum_{\Upsilon \in \mathcal{T}_{i}(\Delta)}\left|\tilde{H}_{i-1}(\Upsilon ; \mathbb{Z})\right|^{2}, \\
\pi_{i} & =\text { product of all nonzero eigenvalues of } L_{\Delta, i-1} .
\end{aligned}
$$

The following formulas relate the tree enumerators $\tau_{i}$ to the linear-algebraic invariants $\pi_{i}$.

Theorem 4.1 (The simplicial matrix-tree theorem) [9] Thm. 1.3] For all $i \leq d$, we have

$$
\pi_{i}=\frac{\tau_{i} \tau_{i-1}}{\left|\tilde{H}_{i-2}(\Delta ; \mathbb{Z})\right|^{2}}
$$

Moreover, if $\Upsilon$ is any spanning tree of $\Delta_{(i-1)}$, then

$$
\tau_{i}=\frac{\left|\tilde{H}_{i-2}(\Delta ; \mathbb{Z})\right|^{2}}{\left|\tilde{H}_{i-2}(\Upsilon ; \mathbb{Z})\right|^{2}} \operatorname{det} \tilde{L}
$$

where $\tilde{L}$ is the reduced Laplacian formed by removing the rows and columns corresponding to $\Upsilon$.

Recall that when $d=1$, the number $\tau_{1}(\Delta)$ is simply the number of spanning trees of the graph $\Delta$, and $\tau_{0}(\Delta)$ is the number of vertices (i.e., 0 -dimensional spanning trees). Therefore, the formulas above specialize to the classical matrix-tree theorem.

Corollary 4.2 Let $i<d$. Suppose that $\tilde{H}_{i-1}(\Delta ; \mathbb{Z})=0$ and that $\Delta$ has an $i$-dimensional spanning tree $\Upsilon$ such that $\tilde{H}_{i-1}(\Upsilon ; \mathbb{Z})=0$. Then the order of the $i$-dimensional critical group is the torsion-weighted number of $(i+1)$-dimensional spanning trees, i.e.,

$$
\left|K_{i}(\Delta)\right|=\tau_{i+1} .
$$

Example 4.3 Returning again to the bipyramid B, recall that 15 is both the number of its spanning trees (Example 3.3) and the order of its 1-dimensional critical group (Example 3.5), in each case because $\operatorname{det} \tilde{L}=15$.

Another formula for the orders of the critical groups of $\Delta$ is as follows. For $0 \leq j \leq d$, denote by $\pi_{j}$ the product of the nonzero eigenvalues of the Laplacian $L_{j-1}^{u d}=\partial_{j} \partial_{j}^{*}$. Then Corollary 4.2 together with [10, Corollary 2.10], implies the following formula for $\left|K_{i}(\Delta)\right|$ as an alternating product:

Corollary 4.4 Under the conditions of Corollary 4.2. for every $i \leq d$, we have

$$
\left|K_{i}(\Delta)\right|=\prod_{j=0}^{i} \pi_{j}^{(-1)^{i-j}} .
$$


The condition that $\Delta$ and $\Upsilon$ be torsion-free is not too restrictive, in the sense that many simplicial complexes of interest in combinatorics (for instance, all shellable complexes) are torsion-free and have torsion-free spanning trees.

Remark 4.5 When every spanning tree of $\Delta$ is torsion-free, the order of the critical group is exactly the number of spanning trees. This is a strong condition on $\Delta$, but it does hold for some complexes notably for simplicial spheres, whose spanning trees are exactly the (contractible) subcomplexes obtained by deleting a single facet. Thus a given explicit bijection between spanning trees and elements of the critical group amounts to an abelian group structure on the set of facets of a simplicial sphere.

Determining the structure of the critical group is not easy, even for very special classes of graphs; see, e.g., [6, 18]. One of the first such results is due to Lorenzini [21, 22] and Merris [24, Example 1(1.4)], who independently noted that the critical group of the cycle graph on $n$ vertices is $\mathbb{Z} / n \mathbb{Z}$, the cyclic group on $n$ elements. Simplicial spheres are the natural generalizations of cycle graphs from a tree-enumeration point of view. In fact, the theorem of Lorenzini and Merris carries over to simplicial spheres in the following way.

Theorem 4.6 Let $\Sigma$ be a d-dimensional simplicial sphere with $n$ facets. Then $K_{d-1}(\Sigma) \cong \mathbb{Z} / n \mathbb{Z}$.

For the proof, see [11].

The condition that $\Sigma$ be a simplicial sphere can be relaxed: in fact, the proof goes through for any $d$-dimensional pseudomanifold $\Sigma$ such that $\tilde{H}_{d-1}(\Sigma ; \mathbb{Z})=0$. On the other hand, if $\Sigma$ is APC in addition to being a pseudomanifold (for example, certain lens spaces—see [17, p. 144]), then it has the rational homology type of either a sphere or a ball (because $\tilde{H}_{d}(\Sigma ; \mathbb{Q})$ is either $\mathbb{Q}$ or 0 ; see [26, p. 24]).

Remark 4.7 Let $\Delta$ be the simplex on vertex set $[n]$, and let $k \leq n$. Kalai [19] proved that $\tau_{k}(\Delta)=$ $n\left(\begin{array}{c}n-2 \\ k\end{array}\right)$ for every $n$ and $k$, generalizing Cayley's formula $n^{n-2}$ for the number of labeled trees on $n$ vertices. Maxwell [23] studied the skew-symmetric matrix

$$
A=\left[\frac{\tilde{\partial}_{\Delta, k}}{-\tilde{\partial}_{\Delta, k+1}^{*}}\right]
$$

where $\tilde{\partial}_{\Delta, k}$ denotes the reduced boundary map obtained from the usual simplicial boundary $\partial_{\Delta, k}$ by deleting the rows corresponding to $(k-1)$-faces containing vertex 1 , and $\tilde{\partial}_{\Delta, k+1}^{*}$ is obtained from $\partial_{\Delta, k+1}^{*}$ by deleting the rows corresponding to $k$-faces not containing vertex 1 . (Note that Maxwell and Kalai use the symbol $I_{r}^{k}(X)$ for what we call $\partial_{\Delta, k}$.)

In particular, Maxwell [23. Prop. 5.4] proved that

$$
\text { coker } A \cong(\mathbb{Z} / n \mathbb{Z})^{\left(\begin{array}{c}
n-2 \\
k
\end{array}\right)} \text {. }
$$

The matrix $A$ is not itself a Laplacian, but is closely related to the Laplacians of $\Delta$. Indeed, Maxwell's result, together with ours, implies that all critical groups of $\Delta$ are direct sums of cyclic groups of order $n$, for the following reasons. We have

$$
A A^{T}=-A^{2}=\left[\frac{\tilde{\partial}_{\Delta, k}}{-\tilde{\partial}_{\Delta, k+1}^{*}}\right]\left[\tilde{\partial}_{\Delta, k}^{*} \mid-\tilde{\partial}_{\Delta, k+1}\right]=\left[\begin{array}{c|c}
\tilde{L}_{k-1}^{u d} & 0 \\
\hline 0 & \tilde{L}_{k+1}^{d u}
\end{array}\right]
$$


where "ud" and "du" stand for "up-down" and "down-up" respectively (see footnote (ii)). Therefore

$$
\begin{aligned}
\operatorname{coker}\left(A A^{T}\right) & \cong \operatorname{coker}\left(\tilde{L}_{k-1}^{u d}\right) \oplus \operatorname{coker}\left(\tilde{L}_{k+1}^{d u}\right) \\
& \cong \operatorname{coker}\left(\tilde{L}_{k-1}^{u d}\right) \oplus \operatorname{coker}\left(\tilde{L}_{k}^{u d}\right) \\
& \cong K_{k-1}(\Delta) \oplus K_{k}(\Delta),
\end{aligned}
$$

where the second step follows from the general fact that $M M^{T}$ and $M^{T} M$ have the same multisets of nonzero eigenvalues for any matrix $M$, and the third step follows from Theorem 3.4 . On the other hand, we have $\operatorname{coker}\left(A A^{T}\right)=\operatorname{coker}\left(-A^{2}\right)=\operatorname{coker}\left(A^{2}\right) \cong(\operatorname{coker} A) \oplus(\operatorname{coker} A)$. It follows from Maxwell's result that the kth critical group of the n-vertex simplex is a direct sum of $\left(\begin{array}{c}n-2 \\ k\end{array}\right)$ copies of $\mathbb{Z} / n \mathbb{Z}$, as desired.

\section{The Critical Group as a Model of Discrete Flow}

In this section, we describe an interpretation of the critical group in terms of flow, analogous to the chipfiring game. By definition of $K_{i}(\Delta)$, its elements may be represented as integer vectors $\mathbf{c}=\left(c_{F}\right)_{F \in \Delta_{i}}$, modulo an equivalence relation given by the Laplacian. These configurations are the analogues of the configurations of chips in the graph case $(i=0)$. When $i=1$, it is natural to interpret $c_{F}$ as a flow along the edge $F$, in the direction given by some predetermined orientation; a negative value on an edge corresponds to flow in the opposite direction. More generally, if $F$ is an $i$-dimensional face, then we can interpret $c_{F}$ as a generalized $i$-flow, again with the understanding that a negative value on a face means a $i$-flow in the opposite orientation. For instance, 2-flow on a triangle represents circulation around the triangle, and a negative 2-flow means to switch between clockwise and counterclockwise.

When $i=1$, the condition $\mathbf{c} \in \operatorname{ker} \partial_{i}$ means that flow neither accumulates nor depletes at any vertex; intuitively, matter is conserved. In general, we call an $i$-flow conservative if it lies in ker $\partial_{i}$. For instance, when $i=2$, the $\partial_{i}$ map converts 2-flow around a single triangle into 1-flow along the three edges of its boundary in the natural way; for a 2 -flow on $\Delta$ to be conservative, the sum of the resulting 1-flows on each edge must cancel out, leaving no net flow along any edge. In general, the sum of (the boundaries of) all the $i$-dimensional flows surrounding an $(i-1)$-dimensional face must cancel out along that face.

That the group $K_{i}(\Delta)$ is a quotient by the image of the Laplacian means that two configurations are equivalent if they differ by an integer linear combination of Laplacians applied to $i$-dimensional faces. This is analogous to the chip-firing game, where configurations are equivalent when it is possible to get from one to the other by a series of chip-firings, each of which corresponds to adding a column vector of the Laplacian. When $i=1$, it is easy to see that firing an edge $e$ (adding the image of its Laplacian to a configuration) corresponds to diverting one unit of flow around each triangle containing $e$ (see Example 5.1). More generally, to fire an $i$-face $F$ means to divert one unit of $i$-flow from $F$ around each $(i+1)$-face containing $F$.

By Theorem 3.4 we may compute the critical group as $\mathbb{Z}^{\Theta}$ modulo the image of the reduced Laplacian. In principle, passing to the reduced Laplacian means ignoring the $i$-flow along each facet of an $i$-dimensional spanning tree $\Upsilon$. In the graph case $(i=0)$, this spanning tree is simply the bank vertex. The higher-dimensional generalization of this statement is that the equivalence class of a configuration $\mathbf{c}$ is determined by the subvector $\left(c_{F}\right)_{F \in \Delta \backslash \Upsilon \text {. }}$.

A remaining open problem is to identify the higher-dimensional "critical configurations", i.e., a set of stable and recurrent configurations that form a set of coset representatives for the critical group. Recall 
that in the chip-firing game, when vertex $i$ fires, every vertex other than $i$ either gains a chip or stays unchanged. Therefore, we can define stability simply by the condition $\mathbf{c}_{i}<\operatorname{deg}(i)$ for every non-bank vertex $i$. On the other hand, when a higher-dimensional face fires, the flow along nearby faces can actually decrease. Therefore, it is not as easy to define stability. For instance, one could try to define stability by the condition that no face can fire without forcing some face (either itself or one of its neighbors) into debt. However, with this definition, there are some examples (such as the 2-skeleton of the tetrahedron) for which some of the cosets of the Laplacian admit more than one critical configuration. Therefore, it is not clear how to choose a canonical set of coset representatives analogous to the critical configurations of the graphic chip-firing game.

Example 5.1 We return once again to the bipyramid B, and its 1-dimensional spanning tree $\Upsilon$ with edges 12, 13, 14, 15. If we pick 1-flows on $\Theta=\Delta_{1} \backslash \Upsilon$ as shown in Figure 2(a) it is easy to compute that we need 1-flows on $\Upsilon$ as shown in Figure 2(b) to make the overall flow 1-conservative. Since Theorem 3.4 implies we can always pick flows on $\Upsilon$ to make the overall flow 1-conservative, we only show flows on $\Theta$ in subsequent diagrams.

If we fire edge 23, we get the configuration shown in Figure 2(c), One unit of flow on edge 23 has been diverted across face 234 to edges 24 and 34, and another unit of flow has been diverted across face 235 to edges 25 and 35. Note that the absolute value of flow on edge 25 has actually decreased, because of its orientation relative to edge 23. If we subsequently fire edge 24 , we get the configuration shown in Figure $2(d)$ One unit of flow on edge 24 has been diverted across face 234 to edges 23 and 34, and another unit of flow has been diverted across face 124 to edges 12 and 14 (and out of the diagram of $\Theta$ ).

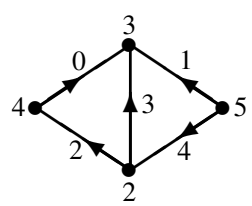

(a)

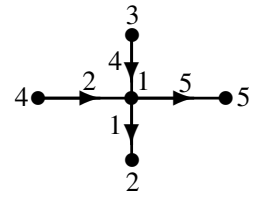

(b)

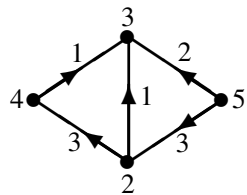

(c)

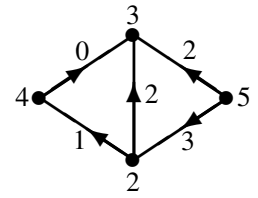

(d)

Fig. 2: Conservative 1-flows and firings

\section{Critical Groups as Chow Groups}

An area for further research is to interpret the higher-dimensional critical groups of a simplicial complex $\Delta$ as simplicial analogues of the Chow groups of an algebraic variety. (For the algebraic geometry background, see, e.g., [16, Appendix A] or [13].) We regard $\Delta$ as the discrete analogue of a $d$-dimensional variety, so that divisors correspond to formal sums of codimension-1 faces. Even more generally, algebraic cycles of dimension $i$ correspond to simplicial $i$-chains. The critical group $K_{i}(\Delta)$ consists of closed $i$-chains modulo conservative flows (in the language of Section 5 is thus analogous to the Chow group of algebraic cycles modulo rational equivalence. This point of view has proved fruitful in the case of graphs [1, 2, 15, 21, 22]. In order to develop this analogy fully, the next step is to define a ring structure on $\bigoplus_{i>0} K_{i}(\Delta)$ with a ring structure analogous to that of the Chow ring. The goal is to define a "critical ring" whose multiplication encodes a simplicial version of intersection theory on $\Delta$. 


\section{References}

[1] Roland Bacher, Pierre de la Harpe, and Tatiana Nagnibeda, The lattice of integral flows and the lattice of integral cuts on a finite graph, Bull. Soc. Math. France 125 (1997), no. 2, 167-198.

[2] Matthew Baker and Serguei Norine, Riemann-Roch and Abel-Jacobi theory on a finite graph, Adv. Math. 215 (2007), no. 2, 766-788.

[3] Norman Biggs, Chip-firing and the critical group of a graph, J. Alg. Combin. 9 (1999), 25-45.

[4] Anders Björner, László Lovász, and Peter Shor, Chip-firing games on graphs, European J. Combin. 12 (1991), no. 4, 283-291.

[5] Ethan Bolker, Simplicial geometry and transportation polytopes, Trans. Amer. Math. Soc. 217 (1976), 121-142.

[6] Hans Christianson and Victor Reiner, The critical group of a threshold graph. Linear Algebra Appl. 349 (2002), 233-244.

[7] Deepak Dhar, Self-organized critical state of sandpile automaton models, Phys. Rev. Lett. 64 (1990), no. $14,1613-1616$.

[8] Jozef Dodziuk and Vijay Kumar Patodi, Riemannian structures and triangulations of manifolds, $J$. Indian Math. Soc. (N.S.) 40 (1976), no. 1-4, 1-52 (1977).

[9] Art M. Duval, Caroline J. Klivans, and Jeremy L. Martin, Simplicial matrix-tree theorems, Trans. Amer. Math. Soc. 361 (2009), no. 11, 6073-6114.

[10] Art M. Duval, Caroline J. Klivans, and Jeremy L. Martin, Cellular spanning trees and Laplacians of cubical complexes, Adv. Appl. Math. 46 (2011), 247-274.

[11] Art M. Duval, Caroline J. Klivans, and Jeremy L. Martin, Critical groups of simplicial complexes, preprint (2011), arXiv:1101.3981

[12] Beno Eckmann, Harmonische Funktionen und Randwertaufgaben in einem Komplex, Comment. Math. Helv. 17 (1945), 240-255.

[13] William Fulton, Intersection Theory, 2nd ed., Springer-Verlag, Berlin, 1998.

[14] Chris Godsil and Gordon Royle, Algebraic Graph Theory, Graduate Texts in Mathematics 207, Springer-Verlag, New York, 2001.

[15] Christian Haase, Gregg Musiker, and Josephine Yu, Linear systems on tropical curves, preprint, arXiv:0909.3685, to appear in Math. Z.

[16] Robin Hartshorne, Algebraic geometry, Graduate Texts in Mathematics, 52, Springer-Verlag, New York-Heidelberg, 1977.

[17] Allen Hatcher, Algebraic Topology. Cambridge University Press, Cambridge, 2002; available online at http: //www.math.cornell.edu/ hatcher/AT/ATpage.html 
[18] Brian Jacobson, Andrew Niedermaier, and Victor Reiner, Critical groups for complete multipartite graphs and Cartesian products of complete graphs, J. Graph Theory 44 (2003), no. 3, 231-250.

[19] Gil Kalai, Enumeration of Q-acyclic simplicial complexes, Israel J. Math. 45 (1983), no. 4, 337351.

[20] Lionel Levine and James Propp, What is... a sandpile?, Notices Amer. Math. Soc. 57 (2010), no. 8, 976-979.

[21] Dino J. Lorenzini, Arithmetical graphs, Math. Ann. 285 (1989), no. 3, 481-501.

[22] Dino J. Lorenzini, A finite group attached to the Laplacian of a graph, Discrete Math. 91 (1991), no. 3, 277-282.

[23] Molly Maxwell, Enumerating bases of self-dual matroids, J. Combin. Theory Ser. A 116 (2009), 351-378.

[24] Russell Merris, Unimodular equivalence of graphs, Lin. Alg. Appl. 173 (1992), 181-189.

[25] Gregg Musiker, The critical groups of a family of graphs and elliptic curves over finite fields, $J$. Algebraic Combin. 30 (2009), no. 2, 255-276.

[26] Richard P. Stanley, Combinatorics and Commutative Algebra, 2nd ed., Birkhäuser, 1996. 